\title{
Image guided robotic interstitial brachytherapy, a new innovative treatment for malignancies
}

\author{
Bhaskar Vishwanathan ${ }^{1, \#, ~ S a n j e e t ~ K u m a r ~ M a n d a l, " \#, ~ R i s h a b h ~ K u m a r,, \# ~ a n d ~ H V ~ R a m p r a k a s h ~}{ }^{2}$ \\ ${ }^{1}$ Department of Radiation Oncology, Vydehi Institute of Medical Sciences \& Research Center, Bengaluru, India \\ ${ }^{2}$ Department of Radiodiagnosis, Vydehi Institute of Medical Sciences \& Research Center, Bengaluru, India \\ \#Contributed equally
}

\begin{abstract}
Introduction: The evolution of brachytherapy has been refined over years, but many of the techniques remain unchanged. The limited utilisation of brachytherapy in comparison to conformal external radiotherapy may be due to its invasive approach, operative risk, technical challenge, time consuming, long learning curve and poor technological advancements. However, there is growing evidence for practicing hypo-fractionated regimes in many solid malignancies. The present article focus on the similar roles of image guided - robotic interstitial high dose rate brachytherapy with hepatocellular carcinoma (HCC) as prototype.

Materials and methods: The robotic brachytherapy safety, commissioning and quality assurance should follow American Association of Physicists in Medicine (AAPM) task group - 43 and the Groupe Européen de Curiethérapie - European Society for Radiotherapy \& Oncology (GEC ESTRO) report of task group 192 recommendations. The patient selection criteria, pre-treatment planning, technique and treatment of image guided - robotic interstitial brachytherapy for liver malignancy are outlined. We used the image guided robotic interstitial high dose rate brachytherapy to treat a large sized paediatric un-resectable HCC; not suitable for transplant/ resection/ RFA and refractory to medical treatment.
\end{abstract}

Results: There were no intra-operative or immediate post-operative complications and at 6 weeks post-procedure assessment, we achieved near complete response with no symptoms.

Conclusions: This is the first study in usage of image based robotic interstitial high dose rate brachytherapy for liver malignancies. The technique described is simple, safe, fast, precise and effective treatment modality for hepatocellular carcinoma. We also emphasis the image guided robotic interstitial brachytherapy as an exciting platform for its similar role to ablate liver metastases or other site malignancies.

\section{Introduction}

The evolution of brachytherapy has been refined over years, but many of the techniques remain unchanged. The inherent benefit to deliver high dose and rapid dose fall of by brachytherapy has been currently well studied only for few sites such as cervix and prostate cancer. The limited utilisation of brachytherapy in comparison to conformal external radiotherapy may be due to its invasive approach, operative risk, technical challenge, time consuming, long learning curve and poor technological advancements. However, there is growing evidence for practicing hypo-fractionated regimes in many solid malignancies such as lung, liver, prostate, pancreas, central nervous system and sarcomas by robotic aided stereotactic radiotherapy. The present article focus on the similar roles of image guided - robotic interstitial high dose rate brachytherapy with hepatocellular carcinoma as prototype.

The Hepatocellular Carcinoma (HCC) is the fifth most common tumor worldwide and the second most common cause of cancer-related death [1]. Male to female predominance is greater than 2:1 with liver cancer, and approximately $83 \%$ of the estimated 782,000 new HCC cases in 2012 occurred in less developed regions of the world, with East and South Asia plus sub-Saharan Africa being the high-incidence regions and Northern Europe and South-Central Asia are the lowincidence regions [2]. Cirrhosis is an important risk factor for HCC and may be caused by chronic viral hepatitis $\mathrm{B}$ or $\mathrm{C}$, alcohol, inherited metabolic diseases such a hemochromatosis or alpha-1-antitrypsin deficiency, and non-alcoholic fatty liver disease. The Barcelona Clinic for Liver Cancer (BCLC) staging and guidelines were last revised in 2011 and are strongly recommended by the American Association for the Study of Liver Disease (AASLD) and the European Association for the Study of Liver (EASL). BCLC guidelines do not endorse subsequent clinical data to practice radiotherapy for HCC. With the establishment of the Model for End stage Liver Disease (MELD) system, five-year survival without HCC therapy, with local tumor ablation, surgical resection and liver transplantation was $15.2 \%, 37.6 \%, 55.5 \%$ and $77.2 \%$ respectively [3]. The low survival rate could be due to advanced stage at presentation, low resectablility rate, high post resection recurrence, poor tumour response and underutilisation of radiotherapy/ combined treatment approaches.

Modern advances in treatment design and delivery have renewed enthusiasm for radiation therapy as an effective local treatment modality for HCC. More recently, the development of Stereotactic

Correspondence to: Sanjeet Kumar Mandal, Department of Radiation Oncology, Vydehi Institute of Medical Sciences \& Research Center, Bengaluru, India, Tel: 080-28410875; Email: sanjeetmandal2020@gmail.com

Key words: robotic, brachytherapy, interstitial, HDR, hepatocellular carcinoma

Received: February 03, 2018; Accepted: February 23, 2018; Published: February 17,2018 
Body Radio-Therapy (SBRT) and charged particle radio-therapy have been explored as a technique minimizing radiation dose to adjacent normal tissues by delivering high doses of radiotherapy in a small number of fractions with high precision. A recent meta-analysis showed the pooled local control rates of SBRT and charged particle therapy to be $87 \%$ and $86 \%$, respectively, and the rates of acute hepatic toxicities of grade $>3$ to be $4.9 \%$ and $3.1 \%$, respectively [4]. Similar to precise SBRT and charged particle radiotherapy, investigators have used computer tomography (CT) guided Interstitial High Dose Rate Brachytherapy to treat unresectable liver tumours which showed increasing median survival to $14-36$ months with $11-20 \%$ mild and $<5 \%$ severe complication rate $[5,6]$. The CT guided iHDRBT for HCC may be an effective alternative modality for non-transplant candidates, unresectable cases and patients unsuitable/ refractory for radiofrequency ablation (RFA), SBRT, Trans-Arterial Chemo-Embolisation (TACE) or sorafenib. However, the precise BT needle insertion has always been a challenge to the radiation oncologist especially for tumors that are deep seated, large in size, multiple, irregular shape, close to major vessel/ hepatobiliary tract/ diaphragm/ pleura/ lung or other normal sensitive structures. We have endeavoured to develop a new advanced technique for simple, safe, fast and precise interstitial brachytherapy under stereotactic robotic guidance called Image Guided - Robotic Interstitial Brachytherapy.

\section{Image guided - robotic interstitial brachytherapy procedure}

\section{Pre-treatment planning}

After selection of a patient for robotic brachytherapy, a pre-plan is developed to estimate the number of brachytherapy catheters, planes \& direction of needle insertion, dose to tumour target and organs at risk. Patient is simulated in supine position with CT simulation isocenter placed at the level of xiphisternum level in mid-body plane. Contrast images in arterial phase at taken with breath hold. Body immobilisation is achieved by vacloc.

\section{Technique}

The robotic interstitial brachytherapy safety, commissioning and quality assurance should follow American Association of Physicists in Medicine (AAPM) task group - 43 and the Groupe Européen de Curiethérapie - European Society for Radiotherapy \& Oncology (GEC ESTRO) report of task group 192 recommendations [7]. The procedure is carried out in the CT scan room under aseptic precautions. The prophylactic use of antibiotic is optional. The patient is placed in supine position on a flat couch with the same CT isocenter as preplanned and then the robotic positioning system integrated to CT machine is mounted. Under general anaesthesia or sedation and intubation as required, a baseline triple phase or arterial contrastCT scan is acquired under induced deep expiratory apnoea and sent to commercially available robotic work station after $1 \mathrm{~mm}$ image reconstruction to improve placement of the brachytherapy catheters. Vacloc can be used to immobilise body as in pre-planning. Preferably, the catheter placement is done along inferio-superior and medio-lateral direction. After selecting the desired target in the lesion, entry point on the skin surface, direction, angulation and needle length on the robotic work station, a $4 \mathrm{~F}$ interstitial steel needle with $6 \mathrm{~F}$ angiographic hydrophilic coated sheath and rigid plastic dilator is attached to the 11$13 \mathrm{G}$ bush and adapter of the robotic positioning arm. Upon position confirmation of the plan, the robot was executed and the robotic arm automatically moved to the designated position over skin. Under induced deep expiratory apnoea, a small $0.5 \mathrm{~cm}$ skin incision is made on the patient skin at the precisely designated by the robot with 11 number surgical blade under local anaesthesia (Lignocaine $2 \%+$ Bupivacaine). The interstitial needle with angiographic sheath attached to robotic arm is introduced through the skin incision to the pre-determined depth carefully. The interstitial needle is removed and replaced by $5-6 \mathrm{~F}$ flexible single leader brachytherapy catheter. The procedure is repeated for the next catheter placement with $3-4 \mathrm{~cm}$ distance apart. CT scan can be used check the proper positioning of the catheter after every 2-3 insertions. Once the robotic brachytherapy procedure is complete, a CT scan is done for planning with or without x-ray marker placed in the catheter and sent to the brachytherapy work station.

\section{Treatment}

The delineation of Clinical Target Volume (CTV) comprising Gross Tumor Volume (GTV) with or without 2-3mm margin and Organs At Risk (OARs) such as remaining normal liver, duodenum, stomach, kidneys, lung and others are done. After the catheter reconstruction, dose prescription of 20-45Gy in 1-3fraction is given and plan optimisation done either by dwell-time or graphical method using commercially available brachytherapy treatment planning software featured with AAPM TG-43 recommendations. The plan is evaluated and approved such that CTV D $90 \geq 100 \%$ would be prescribed dose, liver $2 / 3^{\text {rd }}$ volume receives $<5 \mathrm{~Gy}$, stomach or duodenum D1cc receives $<15 \mathrm{~Gy}$. A typical high-dose rate (HDR) after-loading system featuring a 192 iridium source is used to deliver radiation. The duration of the irradiation ranges from 20-60 minutes depending on the size of the CTV and the activity of iridium source. The tumor volume can be partitioned to perform sequential treatments if the estimated duration of irradiation exceeds this limit. After treatment is delivered, the patient is shifted to CT scan room and the angiographic sheaths with brachytherapy catheters are removed with simultaneous sealing of the tract with gelfoam mixed with iodine contrast. A post removal CT scan is performed to check for proper sealing of the needle tract, any bleed in the liver or peritoneal pouches. The patient is observed in-door for 24-48hours.

\section{Case illustration}

A 7 year old female child presented with abdominal discomfort to our department in July, 2016 with abdominal pain. A triple phase CT scan revealed heterogeneous large mass involving right lobe of liver measuring $11.2 \times 10.4 \times 12.5 \mathrm{~cm}$ with mass effect on adjacent liver parenchyma and right kidney. The portal and hepatic veins were compressed, however no thrombosis. The lesions showed a typical contrast enhancement on arterial phase and wash out in the portal vein suggestive of primary hepatocellular carcinoma. Also, there were few lesion noted in left lobe liver and enlarged celiac node. The Ultrasound guided fine needle cytology of the liver lesion revealed hepatocellular carcinoma. The chest CT scan showed bilateral basal small suspicious nodules with enlarged supraclavicular node. Blood parameters including complete blood count, liver function test, kidney function test and serum electrolytes were normal; but serum AFP was $>3000 \mathrm{ng} / \mathrm{ml}$. The tumour board decision for initial palliative systemic treatment was taken. Accordingly, she received six cycles of cisplatin and doxorubicin from July - December 2016. Assessment at four cycles, the right lobe liver lesion had stable response. After 6weeks of six cycles of chemotherapy, PET-CT scan showed hepatic lesion had increased in size measuring $9.1 \times 9.2 \times 12.3 \mathrm{~cm}$, max SUV 6.3 suggestive of the disease progressed locally, but no extra-hepatic disease. She later received one sitting of trans-arterial embolization to right hepatic artery in Jan-2017. There was partial embolization and patient had prolonged post embolization syndrome. On evaluation at 6 weeks with triple phase CT scan, the right liver lesion showed no response; but mild left lobe hypertrophy (Tables 1 and 2). 
Table 1. Selection of patients with liver cancer for Image Guided - Robotic Interstitial.

\section{Brachytherapy}

- Any number and size of lesions in liver with sufficient residual normal liver.

- No extra-hepatic disease, if definitive therapy is planned.

- BCLC stage O, A, B and C.

- Child Pugh A or selected B.

- Platelet count $>50000 / \mathrm{dl}$, pTT $<50$ s, INR $<1.3$.

- Ascites not a contraindication, though it should minimal or controlled.

- Prior TACE and RAI must be at least 3-4wk.

- Tumour invading vasculature or biliary structures is not a contraindication.

Table 2. Catheter insertion instructions for Image Guided - Robotic Interstitial.

\section{Brachytherapy in liver cancer}

- General anaesthesia or sedation with intubation as required. Pain management should be adequate. Use of vacloc to immobilise body may be required.

- Local anaesthesia should be given percutaneously including liver capsule.

- After baseline arterial contrast CT images are acquired for robotic system, and patient should be immobile.

- Trajectory to target lesion should include a part of normal liver to offer support to angiographic sheath and catheter.

- Needle insertion should be inferior to superior and medial to lateral direction i.e lowermedial then lower-lateral followed by next upper plane.

- Traditional equi-spacing of catheters are not mandatory. Distance between catheters should be generally $3-4 \mathrm{~cm}$.

- For lesion close to lung/pleura, an orbital angulation - rotatory motion along $\mathrm{z}$ axis and caudo-cranial approach - introducing needle from inferior to reach superior target.

- Avoid injury to major vessels - portal vein \& its main branches, common-, right- and left- hepatic arteries.

- Needles passing through bowel / lung-pleura with minimal injury may be acceptable.

- After treatment, the removal of angiographic sheath and catheter should be done with simultaneously track sealing with gel foam mixed with iodine contrast.

Patient underwent robotic interstitial brachytherapy as described above in March-2017, there was no intra-operative complication. A dose of $30 \mathrm{~Gy}$ in 2 fractions was prescribed to CTV; the planned dosimetric parameters for each fraction were CTV D90 - 8.86Gy, liver V66\% - 1.93Gy, stomach D1cc - 2.64Gy, duodenum D1cc- 6.04Gy, kidney right V66\% - 3.6Gy and spinal cord D1cc - 3Gy. The treatment was delivered by HDR - Remote After Loading Brachytherapy machine with in room video monitoring. After removal of catheters, the haemostasis was assured and post- procedure CT scan did not show any collection in liver or abdomen. Child was shifted to PICU, for observation for 24-48 hours. There were no immediate post-operative complications with normal complete blood count, liver function test, kidney function test and serum electrolytes. Patient was discharged with advice on post procedure day- 4 and advised for close follow up. At 6 week post robotic brachytherapy response evaluation, the patient was asymptomatic and whole body PET-CT scan showed - interval regression of the size with increase in necrotic component and near complete resolution of FDG uptake was seen in right lobe liver lesion, now measuring $7.1 \times 5.4 \times 8 \mathrm{~cm}$; $\max$ SUV 2.2; mass effect over adjacent liver parenchyma and kidney were also reduced. Blood parameters including liver and kidney function test were normal; but serum AFP was $>3000 \mathrm{ng} / \mathrm{ml}$. The patient achieved partial response according to mRECIST criteria [8] and near complete metabolic response (Figures 1-3).

\section{Discussion}

Image Guided - Robotic Interstitial Brachytherapy, a new innovative interstitial brachytherapy technique with stereotactic guidance by robotic system has been indigenously developed for the first time to treat malignancies with special emphasis on precision and rapid dose fall off, thereby delivering high dose to tumour target while effectively sparing adjacent normal structures. The robotic brachytherapy was used to treat a large sized paediatric un-resectable hepatocellular carcinoma (HCC); not suitable for transplant and refractory to medical treatment. The technique for robotic brachytherapy described above is simple, safe, fast, precise and effective. At 6 weeks post-procedure assessment, we achieved near complete response with no symptoms. This indigenous robotic brachytherapy system provides an impressive near complete response; and hence has the potential to evolve into a new treatment entity.

The current management of HCC includes surgical resection/ hepatectomy, liver transplantation, thermal or chemical ablation, Trans-Arterial Chemo-Embolization (TACE), and medical treatment. However, the role of radiation therapy for HCC management in the modern era has shifted from palliative to curative with encouraging results and minimum or no liver toxicity. The options for external radiotherapy techniques includes a wide array of $3 \mathrm{D}$ conformal radiotherapy (3DCRT), Intensity Modulated Radiotherapy (IMRT), SBRT, Charged Particle Radiotherapy, Trans-Arterial RadioEmbolization (TARE) or Brachytherapy using iodine seeds with local tumour control of $75-100 \%$, one year survival of $55-75 \%$ and severe toxicity were reported in up to $30-40 \%[9,10]$. The role of interstitial radiotherapy has been an area of active research in last two decades. In the past, two similar approaches have been described for liver brachytherapy namely seldinger catheter replacement technique and proguide round needle technique with mean local tumour control of $88-100 \%$ at lyear follow up and severe toxicity occurred in less than $5 \%$ of patient $[5,6]$. But there has been a challenge to use these techniques amongst radiation oncologists due to the complex technique, lack of precision in brachytherapy catheter insertion and placement,

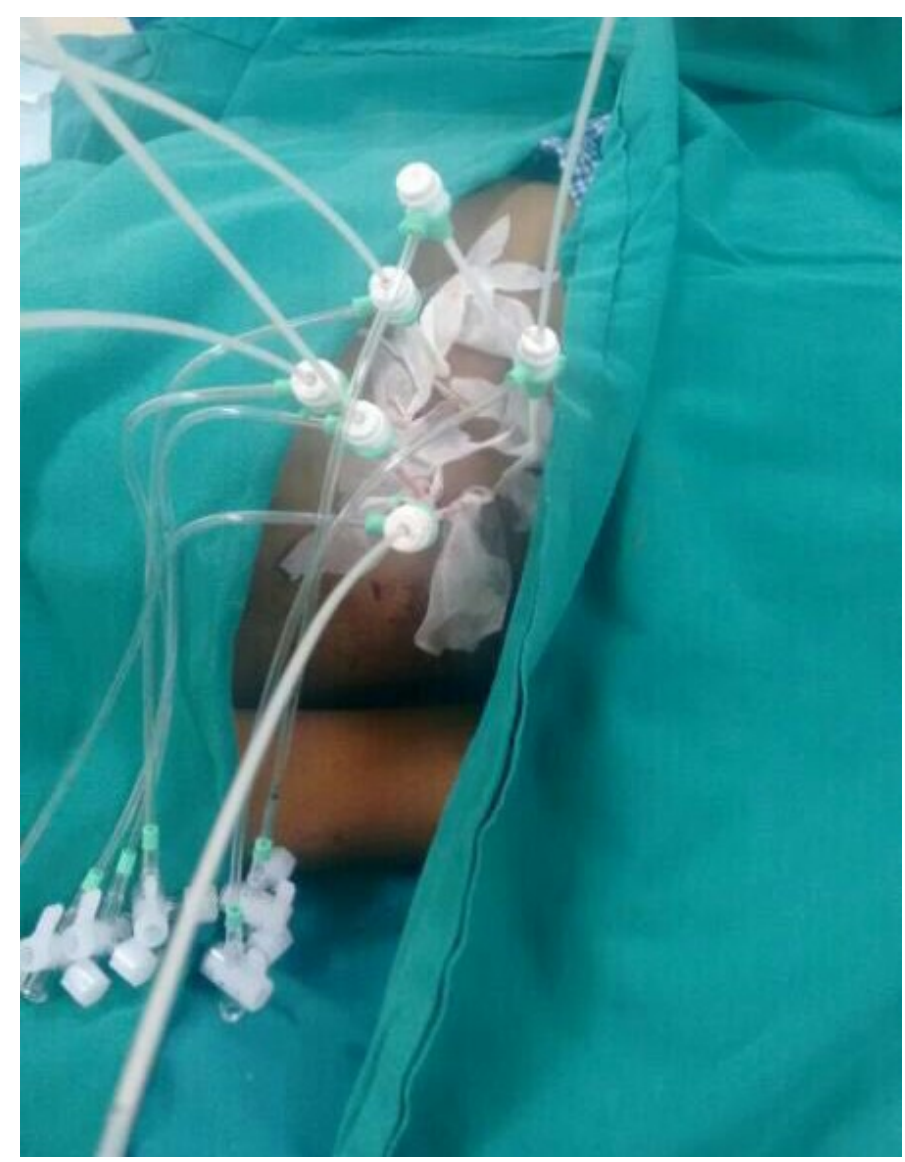

Figure 1. Brachytherapy Procedure. 


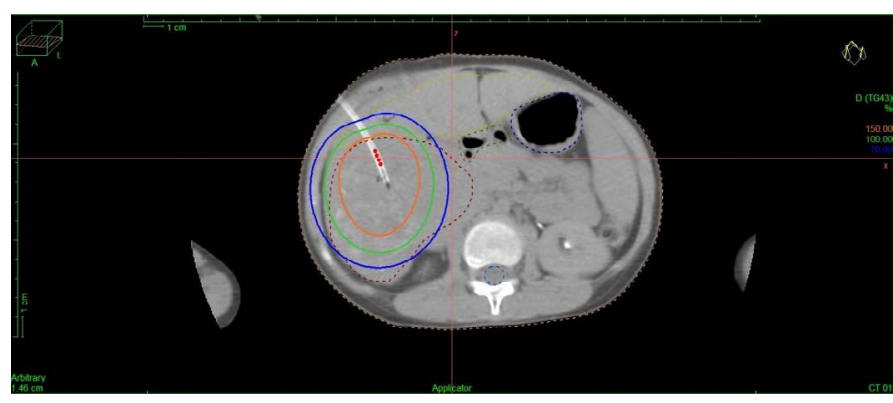

Figure 2. Brachytherapy Treatment Planning.

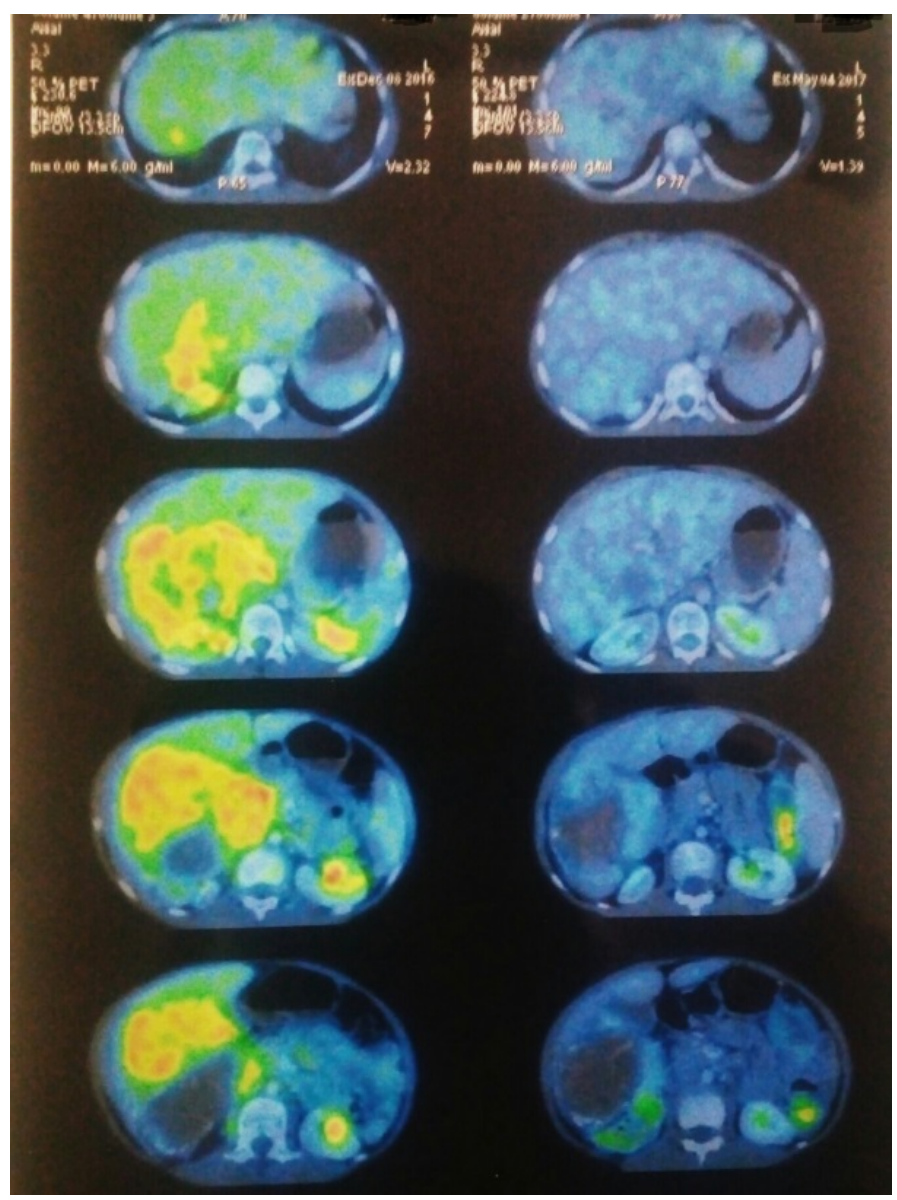

Figure 3. Brachytherapy Treatment Response.

laborious, time consuming and fear of post procedure complications. The Image Guided Robotic Interstitial Brachytherapy technique described above is simple, safe, fast and with sub-millimetre precision for catheter insertion; and also adheres to recommendations of AAPM GEC ESTRO task group 192. The inherent property of brachytherapy i.e., to deliver a high dose to tumour target and rapid dose fall off to spare sensitive normal tissue has been benefitted by integration of the robotic technology. The robotic brachytherapy has been previously used in the treatment of prostate carcinoma and melanoma. The robotic brachytherapy for the treatment of hepatocellular carcinoma was done for the first time and has shown excellent tumour response with no toxicity. The robotic brachytherapy is an evolving technology in cancer therapeutics. We also emphasis that image guided robotic interstitial high dose rate brachytherapy can be similarly used to ablate liver metastases or other site malignancies.

\section{Conclusions}

To the best of our knowledge and literature review, this is the first study in usage of image based robotic interstitial high dose rate brachytherapy for liver malignancies. The technique described is simple, safe, fast, precise and effective treatment modality for hepatocellular carcinoma. We also emphasis the image guided robotic interstitial brachytherapy as an exciting platform for its similar role to ablate liver metastases or other site malignancies.

\section{Conflict of interest}

\section{Nil}

\section{References}

1. Ferlay J, Soerjomataram I, ErvikM (2014) Cancer Incidence and Mortality Worldwide: IARC Cancer Base No. 11. International Agency for Research on Cancer, Lyon, France.

2. Choo SP, Tan WL, Goh BK, Tai WM, Zhu AX (2016) Comparison of hepatocellular carcinoma in Eastern versus Western populations. Cancer.

3. Wong RJ, Devaki P, Nguyen L, Cheung R, Cho-Phan C, et al. (2014) Increased longterm survival among patients with hepatocellular carcinoma after implementation of Model for End-stage Liver Disease score. Clin Gastroenterol Hepatol 12: 1534-1540.

4. Qi WX, Fu S, Zhang Q, Guo XM (2015) Charged particle therapy versus photon therapy for patients with hepatocellular carcinoma: a systematic review and metaanalysis. Radiother Oncol 114: 289-295.

5. Tselis N, Chatzikonstantinou G, Kolotas C (2012) Hypofractionated accelerated computed tomography-guided interstitial high-dose-rate brachytherapy for liver malignancies. Brachytherapy 11: 507-514.

6. Ricke J, Wust P, Wieners G (2004) Liver malignancies: CT-guided interstitial brachytherapy in patients with unfavorable lesions for thermal ablation. $J$ Vasc Interv Radiol 15: 1279-1286.

7. Podder TK, Beaulieu L, Cormack RA (2014) AAPM and GEC-ESTRO guidelines for image-guided robotic brachytherapy: report of Task Group 192. Med Phys 41: 101-150.

8. Eisenhauer EA, Therasse P, Bogaerts J (2009) New response evaluation criteria in solid tumours: Revised RECIST guideline (version 1.1). Eur J Cancer 45: 228-247.

9. Bujold A, Massey CA, Kim JJ (2013) Sequential phase I and II trials of stereotactic body radiotherapy for locally advanced hepatocellular carcinoma. J Clin Oncol 13: 1631-1639.

10. Lasley FD, Mannina EM, Johnson CS (2015) Treatment variables related to liver toxicity in patients with hepatocellular carcinoma, Child-Pugh class A and B enrolled in a phase 1-2 trial of stereotactic body radiation therapy. Pract Radiat Oncol 5: e443-9.

Copyright: (C2018 Vishwanathan B. This is an open-access article distributed under the terms of the Creative Commons Attribution License, which permits unrestricted use, distribution, and reproduction in any medium, provided the original author and source are credited. 